\title{
Kompetenzen vor Inhalte: Die Kleine Reihe Hochschuldidaktik Politik
}

\author{
Klaus Dingwerth $\cdot$ Robert Kordts-Freudinger
}

(C) The Editor(s) and the Author(s) 2019

\section{Einleitung}

Tut sich da etwas in der politikwissenschaftlichen Lehre? Knapp 20 Jahre nach der Bologna-Reform hat der Wochenschau Verlag kürzlich die Kleine Reihe Hochschuldidaktik Politik auf den Markt gebracht. Herausgegeben wird die Reihe von den Sprecherinnen und Sprechern des Arbeitskreises Hochschullehre der Deutschen Vereinigung für Politikwissenschaft (DVPW). Im Verlagsprogramm ergänzt sie zum einen die für den Politikunterricht konzipierte Reihe zur politischen Bildung. Zum anderen weitet sie die disziplinär ausgerichtete Auseinandersetzung mit hochschuldidaktischen Fragen, die der Wochenschau Verlag bereits für die Geschichtswissenschaften vorgelegt hatte, nunmehr auf die Politikwissenschaft aus. Dort trifft sie auf einen Zeitgeist, der auch sonst eher forschungszentrierte Fachorgane wie die Politische Vierteljahresschrift (PVS, in der Dezemberausgabe 2018) ausführlich über die Lehre reflektieren lässt. Und sie trifft auf eine junge Generation Lehrender, für die hochschuldidaktische Aus- und Weiterbildungen - mal aus Pflicht, mal aus Interesse - zunehmend zur Norm werden, die durch ebendiese Aus- und Weiterbildungen aber auch offen für den Austausch über Fragen der guten Lehre sind.

Nun kann man sich fragen, warum denn eine Hochschuldidaktik überhaupt disziplinären Grenzen folgen sollte. Ist die Vermittlung politikwissenschaftlicher Inhalte denn derart spezifisch, dass man die Vorgaben der kompetenzorientierten Lehre (Gloe, 2018), des Prüfens (Stykow, 2018), des wissenschaftlichen Schreibens (Cron-

\footnotetext{
K. Dingwerth $(\square)$

SEPS-HSG, Büro 52-5036, Universität St. Gallen (HSG), Müller,-Friedberg-Strasse 6/8, 9000 St.

Gallen, Schweiz

E-Mail: klaus.dingwerth@unisg.ch

R. Kordts-Freudinger

IWP - Institut für Wirtschaftspädagogik, IWP-HSG, Büro 3-210, Universität St. Gallen (HSG),

Dufourstrasse 40a, 9000 St. Gallen, Schweiz

E-Mail: robert.kordts-freudinger@unisg.ch
} 
qvist, 2018) oder des forschenden Lernens (Freise, 2018) eigens für diese Disziplin durchdeklinieren müsste? Die vier Bände deuten Antworten auf diese Frage mitunter an, wählen aber letztlich einen pragmatischen Zugang. Denn ein klarer Vorzug der Reihe ergibt sich für ihre Rezipienten gerade daraus, dass sie den lehrenden Kolleginnen den Einstieg in die Materie durch die Fokussierung auf ihre Disziplin erleichtert. Insbesondere die zahlreichen Beispiele holen Politikwissenschaftlerinnen dort ab, wo sie sich im Lehralltag schon längst befinden.

Spannend ist dabei, dass auch drei der vier der Autorinnen nicht primär Fachdidaktikerinnen sind, sondern ihre eigene Lehrpraxis unter Anleitung theoretischer Ideen aus der Hochschuldidaktik reflektieren. Das ist insofern neu, als didaktische Fragen bislang eher an Lehrstühlen für Politische Bildung/Didaktik verhandelt wurden, die in den politikwissenschaftlichen Instituten nicht immer zum disziplinären Kern gezählt werden. In diesem Sinne spiegelt bereits die Veröffentlichung der Reihe die Wiederentdeckung der Hochschuldidaktik in der Politikwissenschaft. Weiter interpretiert, kann die Reihe auch als Startpunkt für das auch im deutschsprachigen Raum diskutierte Scholarship of Teaching and Learning (vgl. Huber, 2011) dienen, denn sie zeigt auf, wie politikwissenschaftsspezifische Reflexionen über Hochschullehre aussehen können.

Die vier Bände, mit denen die Reihe startet, sind allesamt knapp - die ungefähr 50 Seiten lassen sich jeweils in anderthalb Stunden lesen. Dennoch geben alle vier Bände einen guten ersten Einblick in ihr Thema, den sie zumeist mit Beispielen aus der eigenen Lehrpraxis sowie - angesichts der Kürze der Bände besonders wertvoll - mit Hinweisen auf weitere Literatur ergänzen. Die Themenauswahl ist dabei plausibel. Während der Band zur Kompetenzorientierung das Paradigma vorgibt, in dem sich auch die anderen Bände verorten, deckt insbesondere der Band zum Prüfen einen Bereich ab, der prinzipiell alle (verantwortlich) Lehrenden betrifft. Die Bände zum wissenschaftlichen Schreiben und zum forschenden Lehren sind insofern eher ergänzend. Hier können Lehrende freier entscheiden, ob und wie stark sie sich diese Ziele bzw. Formate in ihrer eigenen Lehre zu eigen machen möchten.

Dass die ersten vier Bände noch Lücken lassen, versteht sich. So kommt das Lehrformat der Vorlesung in allen vier Bänden selten vor. Michael Gloes Band lässt zwar offen, ob es sie in der kompetenzorientierten Lehre überhaupt noch geben darf. Aufgrund ihres günstigen Betreuungsschlüssels werden sie für viele Studiengänge aber wohl relevant bleiben. Auch ein Band zur Form des flipped classroom (vgl. dazu etwa Jenkins, 2015) oder zum problembasierten oder -orientierten Lernen wäre wünschenswert. Zudem könnte ein Band, der die Gestaltung politikwissenschaftlicher Curricula oder Studiengänge hochschuldidaktisch reflektiert und begleitet, für Praktikerinnen hilfreich sein. Er könnte auch noch stärker ins Bewusstsein bringen, was in den vorliegenden Bänden immer wieder einmal aufscheint, aber noch nicht systematisch behandelt wird: nämlich, dass gute Hochschullehre nicht nur eine individuelle Aufgabe der Lehrenden, sondern immer auch eine Gemeinschaftsaufgabe der jeweiligen Institute ist. 


\section{Kompetenzorientierung in der politikwissenschaftlichen Hochschullehre}

Michael Gloes Band vermittelt einen Überblick über die Grundidee der kompetenzorientierten Lehre. Seit Bologna stehe nicht mehr im Vordergrund, ,,welches Wissen erworben werden soll“, sondern ,welche Kompetenzen die Lernenden selbst entwickeln sollen“. Kompetenzen umfassen dabei einerseits „Disposition[en] zum Einsatz von Wissen, Fähigkeiten“, andererseits „Fertigkeiten (...) zur Bewältigung von Aufgaben oder Situationen“, die erlernt oder erworben und durch Üben verbessert werden (Gloe, 2018, 7-8).

Gloe macht deutlich, dass die Kompetenzorientierung letztlich ganze Studiengänge betrifft. Da hier in den vergangenen 20 Jahren recht wenig passiert sei, fordert er die politikwissenschaftlichen Institute auf, sich regelmäßig darüber zu verständigen, welche kognitiven, sozialen und motivationalen Kompetenzen die Studierenden in ihren Studiengängen überhaupt erlernen sollen. Um mögliche Antworten zu sortieren, nennt er Kompetenzen im Bereich des wissenschaftlichen Arbeitens, Analyseund Urteilskompetenzen, sprachliche und schriftliche Kompetenzen und - hier verbirgt sich dann möglicherweise doch eine spezifisch politikwissenschaftliche Herausforderung - „Demokratiekompetenzen“. An anderer Stelle folgen Lernstrategien, Argumentationsfähigkeit, die Fähigkeit zu interdisziplinärem Denken oder die Fähigkeit zu kritischem Denken, die der Autor noch einmal in ,kritisches Hinterfragen von Aussagen, das logische Schlussfolgern, die schlüssige Begründung von Behauptungen und die Vermeidung von Übergeneralisierung und typischen Denkfehlern“ aufschlüsselt (Gloe, 2018, 17).

Gleich, worauf der Fokus am Ende nun genau liegen wird, gilt: Passives Lernen „[ermöglicht] kaum einen Kompetenzerwerb“ (Gloe, 2018, 32, vgl. auch ebd., 12). Studierende, die Kompetenzen statt Wissen erwerben sollen, müssen solche Kompetenzen folglich im Studium einüben können. Zum Ausgangspunkt für Lernprozesse werden somit Aufgaben. So lässt sich Gloes Argumentation etwas verkürzt auf den einfachen Aufruf zuspitzen: Überlegt Euch weniger, was Ihr den Studierenden erzählt - überlegt Euch lieber, welche Aufgaben Ihr ihnen stellt!

Daraus leiten sich zwei wichtige Konsequenzen ab. Weil das Training vor allem dann wirksam sein dürfte, wenn die Übenden auch Rückmeldungen zu ihren Lernfortschritten erhalten, müssen die Lehrenden in das Feedback investieren. Das nimmt Ressourcen in Anspruch. Und weil Kompetenzen zwar an inhaltlichen Fragen ausgebildet werden, ihr Einüben aber einige Zeit in Anspruch nimmt, kann inhaltliche Vollständigkeit nicht mehr als Leitbild dienen - ,legt man in seinen Seminaren den Fokus auf die Förderung von Kompetenzen, so muss man sich auch vom unrealistischen Anspruch einer umfassenden Behandlung von Inhalten verabschieden“ (Gloe 2018, 31, unsere Hervorhebung). Hier wären Beispiele entsprechender Abwägungen aus der eigenen Lehrpraxis spannend.

Was leistet der Band? Aus Sicht eines politikwissenschaftlich Lehrenden bietet er zunächst eine sehr lesbare und auch lesenswerte Einführung in die Grundidee, die im Zuge zahlreicher Reformen der vergangenen Jahre und Jahrzehnte so langsam in der Praxis der politikwissenschaftlichen Lehre ankommt. Dem didaktisch ungeübten Auge liefert er dabei einige wertvolle Ressourcen, um die eigene Lehr- 
praxis zu reflektieren und das eigene Rollenbild zu hinterfragen. Freilich erscheint einem dabei Weniges disziplinspezifisch - die Politikwissenschaft im Titel ist insofern eine kleine Mogelei. Zudem ist an der Kompetenzorientierung längst nicht alles neu. So konnten (und mussten) auch in den alten Magisterstudiengängen, in denen viele Lehrende selbst noch studiert haben dürften, Kompetenzen erworben werden. Das galt insbesondere für die vorlesungsfreie Zeit, in der die zahlreich zu verfassenden Seminararbeiten gleich für mehrere Wochen intensive Gelegenheiten boten zu planen, zu recherchieren, zu lesen, zu sortieren, zu denken, zu schreiben, zu irren, mit Ambivalenzen und Widersprüchen umzugehen und Projekte dann doch - meistens - irgendwie zu einem pragmatischen Abschluss zu bringen. Neu ist also in erster Linie, dass der Kompetenzerwerb auch während der Vorlesungszeit und im Seminarraum der Wissensvermittlung den Rang ablaufen soll. Das macht Sinn aber eben vor allem dann, wenn die vorlesungsfreie Zeit nicht im Gegenzug vom wissenschaftlichen Kompetenzerwerb entleert und für den Erwerb (vermeintlich) praxisrelevanter Kompetenzen in immer mehr Praktika freigeräumt wird.

Aus Sicht eines (mit mehreren Disziplinen arbeitenden) Hochschuldidaktikers bringt Gloe angesichts der genannten allgemeinen thematischen Ausrichtung durchaus Redundantes. Man kann sich also fragen, was ihn jenseits des politikwissenschaftlichen Titels von allgemeinen hochschuldidaktischen Einführungs-Werken unterscheidet. Bezogen auf die Behandlung der Lernziele kommt erschwerend hinzu, dass die Taxonomie nach Bloom als einzige dargestellt wird, obwohl zum einen Aktualisierungen dieses Modells (z. B. nach Anderson \& Krathwol, 2011) als auch alternative Konzeptionen existieren. Positiv stehen dem die gut erfassbaren Zusammenfassungen am Ende jedes Kapitels gegenüber. Sie erlauben es den Leserinnen, sich einfach und schnell einen Überblick über die behandelten Themengebiete zu verschaffen.

\section{Prüfen in politikwissenschaftlichen Studiengängen}

Der stärkste Band der Reihe ist Petra Stykows Auseinandersetzung mit dem Prüfen. „Alle tun es, keiner mag es“, beginnt die Autorin ihre kritische Reflexion der eigenen und in ihrem disziplinären Umfeld beobachteten Praxis des Prüfens (Stykow, 2018, 7). Der Band ist überzeugend im kompetenzorientierten Paradigma verortet; die Spannungen zwischen dem didaktisch gewünschten Fokus auf Kompetenzerwerb und dem hochschulpolitisch notwendigen Erfordernis der Benotung legt die Autorin dabei offen.

Inhaltlich unterscheidet der Band zunächst zwischen Prüfen, Bewerten und Benoten, benennt die Spezifika kompetenzorientierten Prüfens - das eher formativ als summativ sein wird - und geht auf Qualitätsmerkmale von Prüfungen ein. AnschlieBend diskutiert die Autorin die „Prüfungsplanung als Lehrplanung“. Denn wenn Prüfen vor allem zum Lernen beitragen soll, müssen die Prüfungsformate und -aufgaben am Anfang stehen und so gewählt sein, dass sie das Lernen besonders gut unterstützen. Den Abschluss bilden differenzierte Reflexionen von Prüfungsaufgaben, Bewertungskriterien und Prüfungssituationen sowie der Praxis der Notenbildung. 
Vor allem die letztgenannten Kapitel enthalten dabei eine Fülle konkreter Beispiele aus politikwissenschaftlichen Lehrveranstaltungen.

Stykows Ziel ist dabei, dass Studierende Prüfen und Lernen als zusammenhängend erfahren können - das Prüfen soll das Lernen ,,diagnostisch begleiten und fördern“ (Stykow, 2018, 10). Das gelingt vor allem dann, wenn das formative Prüfen als assessment for learning im Vordergrund steht und das summative Prüfen am Ende des Lernprozesses - als ein assessment of learning - nur noch das Erreichen vorab definierter Kompetenzniveaus beglaubigt. Stykow $(2018,12)$ macht aber auch deutlich, dass dort, wo Lernleistungen benotet werden, ,sich das formative Potenzial einer Prüfung nicht entfalten kann“. Der Idealfall sieht für die Autorin demnach so aus: „Die Studierenden bearbeiten ein auch sie interessierendes reales oder realitätsnahes Problem, das politikwissenschaftlich relevant ist und in dem Sinne ill-defined ist, dass es keine ,gesicherte Lösung ' gibt. Dabei setzen sie Wissen und Können ein, das sie in der Lehrveranstaltung erworben haben“ (Stykow, 2018, 23, Herv. i. Orig.).

Die Bearbeitung der Aufgabe wird dann selbst zum Lernprozess, der - anders als noch bei der guten alten Seminararbeit - zusätzlich durch studentisches oder von den Lehrenden zu leistendes Feedback zu definierten Zwischenschritten sowie durch die studentische Reflexion der eigenen Lernstrategien unterstützt wird. Insbesondere vor diesem Hintergrund diskutiert Stykow neben den klassischen Prüfungsformaten auch Lernportfolios, bei denen Protokollnotizen zum Feedback und nachfolgende Textrevisionen ebenfalls zum Teil der Aufgabenstellung werden. Als Zwischenform, die zwischen formativem Anspruch und summativer Notwendigkeit vermitteln kann, erwähnt sie auch das „Hausarbeitsportfolio“.

Politikwissenschaftlich Lehrende profitieren dabei unter anderem davon, dass die Autorin Prüfungsformate breit anspricht, zahlreiche Aspekte des Prüfens sorgfältig einordnet und Spannungen wie Herausforderungen ehrlich, engagiert und stets differenziert erörtert. So behandelt sie beispielsweise mündliche Prüfungen nicht als eigenes Format, sondern als Prüfungssituation. Dies erst erlaubt ihr, Wege des Umgangs mit der oft als unangenehm empfundenen Machtasymmetrie mündlicher Prüfungen zu erörtern. Auch dass bei offenen Prüfungsfragen Bewertungen und Benotungen trotz allen Objektivitätsscheins immer eine subjektive Komponente haben, beschäftigt Stykow. Auflösen kann sie dieses Problem nicht, begegnen will sie ihm aber dadurch, dass Lehrende auch über ihre eigenen Erfahrungen als Autorinnen oder Gutachter sprechen und dass wir die Fähigkeit, mit äußerst unterschiedlichen Rückmeldungen umzugehen, ebenfalls als eine Kompetenz begreifen, die für politikwissenschaftliche Arbeitsfelder wichtig ist. Für die eigene Prüfungspraxis finden sich zudem etliche Anregungen. Insbesondere das Kernargument, dass Prüfungen nicht das notwendige Übel am Ende der Lehrveranstaltung sein müssen, sondern im Gegenteil zu einer im Sinne eines constructive alignment verstandenen und das Lernen erst ermöglichenden Idee am Anfang der Veranstaltungskonzeption werden können, macht Mut und hilft, den Sinn für gutes Prüfen zu schärfen.

Aus Sicht des Hochschuldidaktikers fällt überdies die Fundiertheit und analytische Durchdringung des Textes aus. Obwohl - oder gerade weil? - die Autorin auf der Grundlage eigener Erfahrungen Stellung bezieht, behandelt sie das aktuelle Paradigma des Kompetenzorientierten Prüfens gut fundiert. Zusätzlich hat besonders dieser Band dank der im Text enthaltenen Aufgaben das Potential, bei den 
Lesern eine tiefergehende Reflexion zu bewirken. Aus allgemeindidaktischer Sicht ließe sich zwar kritisieren, dass einige der von der Autorin genannten Beispielformulierungen für prüfungsorientierende Lernziele (z. B. „kennen ...“, Stykow, 2018, 21) nicht ihren eigenen Ansprüchen genügten, andererseits zeigt vielleicht genau dieser Ansatz, dass auch Prüfen immer work in progress ist. Zusammengenommen wirkt der Band von Petra Stykow damit am stärksten wissenschaftlich fundiert und stellungnehmend; den Anforderungen des oben genannten Scholarship of Teaching and Learning kommt er so am nächsten.

\section{Wissenschaftliches Schreiben in der politikwissenschaftlichen Hochschullehre}

Lasse Cronqvists Band nimmt seinen Ausgang in der Beobachtung, dass politikwissenschaftliche Studiengänge zu wenig Gelegenheit geben, das Schreiben regelmäßig zu üben. Dies scheint ihm vor allem deshalb bedenklich, weil ,das Schreiben (...) die wesentliche Form der Präsentation wissenschaftlicher Fortschritte [ist]“ (Cronqvist, 2018, 5). Wie alle Kompetenzen fällt aber auch die Schreibkompetenz nicht vom Himmel - der Autor schreibt hier zurecht gegen einen verbreiteten und für Lehrende vergleichsweise bequemen Begabungsmythos an. Stattdessen kann und muss das wissenschaftliche Schreiben durch regelmäßiges Training erlernt werden. „Schreibübungen“, schreibt der Autor entsprechend, ,dienen durch entsprechendes Feedback dazu, einen Reflexionsprozess über das eigene Schreiben zu unterstützen und somit den Einstieg in das wissenschaftliche Schreiben zu erleichtern" (Cronqvist, 2018, 13).

Allerdings bleibt in Cronqvists Band eine wichtige Unterscheidung im Hintergrund. So kann politikwissenschaftlicher Unterricht einerseits das Schreiben selbst als Kompetenz bei Studierenden fördern. Dann ist das Schreiben ein Zweck, auf den wir den Unterricht ausrichten. Gleichzeitig kann das Schreiben aber auch verwendet werden, um andere Kompetenzen zu fördern. Dann wird Schreiben ein didaktisches Mittel zum Zweck. Vor dem Hintergrund des die Reihe einenden Paradigmas wäre eine Diskussion, die sich diese Unterscheidung für die Strukturierung und Reflexion zentraler Inhalte stärker zu eigen macht, wertvoll.

Hinsichtlich der konkreten Mittel zur Förderung der Schreibfertigkeiten unterscheidet Cronqvist zwischen Essays und Formen des „Schreibdenkens“. Seine Erläuterungen zum Essay gehen dabei leider kaum in die Tiefe und bieten für Lehrende wenig Neues. Was wir durch Essays und Seminararbeiten fördern, an welche Voraussetzungen diese Förderung jeweils gekoppelt ist und welche typischen Denkfehler und im Lehrbetrieb verankerten Routinen den Kompetenzerwerb behindern, erfährt man hier nicht; auch die theoretische Reflexion im Lichte der Fachdidaktik bleibt weitgehend aus.

Anders bei den Überlegungen zum „Schreibdenken“: Da das Format selbst für viele Kollegen unkonventionell ist, ist bereits seine Behandlung für politikwissenschaftlich Lehrende wertvoll. Im Wesentlichen dient das Schreibdenken dazu, den Frontalvortrag zum Seminareinstieg durch eine auf die Seminarlektüre bezogene Schreibübung zu ersetzen. Dabei gibt die Dozentin den Studierenden kurz - in 
Cronqvists Beispiel fünf Minuten - Zeit, um basierend auf der Vorbereitungslektüre wesentliche Gedanken zu einer vorgegebenen Frage oder einem für die Sitzung zentralen Begriff zu sammeln und strukturieren. Vor der Diskussion im Plenum bekommen die Studierenden anschließend alleine oder im Austausch miteinander ein paar weitere Minuten, um die Ergebnisse ihres „Schreibdenkens“ zu reflektieren und sie bei Bedarf zu ergänzen. Aktivierung und Inklusivität sind hier gegenüber dem Einführungsvortrag höher; Lesemotivation und -disziplin dürften steigen; zudem gibt die Übung den Studierenden und Dozierenden genauere Auskunft darüber, was sie (nicht) verstanden haben. Cronqvists Erläuterungen zu Sinn und Zweck sind hier durchweg hilfreich, und seine Praxisbeispiele motivieren zum Nachdenken darüber, wie die Grundideen in die eigene Lehre passen könnten.

Aus hochschuldidaktischer Perspektive fällt allerdings auf, dass Cronqvist wenige weiterführende Quellen anführt, obwohl diese an einigen Stellen sinnvoll wären, so z. B. Verweise auf das Modell des Constructive Alignement (vgl. Biggs \& Tang, 2011). Die gut ausgebaute englischsprachige Hochschuldidaktik wird ebenfalls nicht zitiert, und schließlich fällt auch die Form im Vergleich zu den anderen Bänden der Reihe schwächer aus. Obwohl der Band vom Schreiben handelt, wartet der Autor oft mit komplexen, langen und im Passiv formulierten Sätzen auf, die die Lektüre erschweren.

\section{Forschendes Lernen in der politikwissenschaftlichen Hochschullehre}

Matthias Freises Band beginnt mit einer Verheißung: Forschendes Lernen bedeute zwar „,mehr Aufwand“, dafür aber, ,richtig angewendet - einen Gewinn“ (Freise, 2018, 5), und zwar, neben dem Kompetenzzuwachs der Studierenden, für die eigene Forschung der Lehrenden! Wie forschendes Lernen in der Politikwissenschaft angewendet werden kann, dies legt er in fünf Kapiteln dar, wobei deduktiv vom allgemeinen Prinzip immer stärker auf spezifische Bedingungen eingegangen wird, was schließlich in einer Checkliste Forschendes Lernen kulminiert.

Freise bezieht sich im allgemeinen Teil auf Arbeiten Ludwig Hubers und leitet zum allgemeinen Ablauf des Forschenden Lernens über. Schon hier nimmt er den aus dem forschenden Zugang zum Lernen erwachsenen Anspruch ernst und beleuchtet das Thema auch aus einer forschenden Perspektive, hier anhand von Veröffentlichungen zu Rahmenbedingungen und Wirksamkeit forschenden Lernens. Unter anderem erläutert er, dass forschendes Lernen vom Wissensstand der Studierenden abhängt und damit, so ließe sich verallgemeinern, wie alle anderen didaktischen Konzepte kein magisches Mittel darstellt; Planung von Lehre aufgrund didaktischer Konzepte erfordert kritische Auseinandersetzung auch mit diesen Konzepten - eine wichtige Erkenntnis angesichts auch aktuell virulenter (pseudo-)wissenschaftlicher Heilsversprechen in der Hochschullehre!

Bevor der Autor Hinweise zur konkreten Umsetzung forschenden Lernens in der Politikwissenschaft gibt, erschließt er den Leserinnen mit einer persönlich und gut nachvollziehbar kommentierten Auswahl weiterführender Quellen und Veröffentlichungsorganen weitere hochschuldidaktische Ressourcen zum Thema. Der prakti- 
sche Teil wird auch anhand von Themen behandelt, die zu selten zum Kerngeschäft der Lehre zählen, so z. B. Prüfen und Evaluation.

Aus der Lehrendenperspektive sind auch hier wieder die zahlreichen Beispiele, Verweise auf Praxisumsetzungen und Checklisten hilfreich; die dazugehörigen Reflexionen vermitteln ein differenziertes Bild und geben, bei aller Begeisterung für das Lehrformat, auch der Diskussion seiner Fallstricke und erforderlichen Ressourcen Raum. Aus Programmleiterperspektive stellt sich allerdings die Frage der curricularen Einbindung des studentischen Forschens. So bleibt zum einen offen, in welchem Studienabschnitt das studentische Forschen seine Wirkungen am besten entfalten kann: Am Anfang, um die Forscherinnenperspektive frühzeitig zu erleben und damit ein wichtiges Ziel des Studiums zu Beginn erfahrbar zu machen (was Freise wohl eher nicht unterstützen würde)? Kurz vor Schluss, als Vorbereitung auf die Abschlussarbeit? Oder gar beides? Das führt zum anderen zur Frage danach, wie viel studentisches Forschen einem Studiengang (noch) zugutekommt: Ist das einmalige Einüben eher Minimalanforderung oder im Sinne Kompetenzorientierung bereits genug? Ist mehr studentisches Forschen immer besser - oder müssen politikwissenschaftliche Studiengänge, die ganz auf studentisches Forschen setzen, in wesentlichen Bereichen Kompetenzeinbußen in Kauf nehmen?

Interessant ist zudem Freises Hinweis zum Mehrwert der Kooperation mit Dritten. Denn wenn das angeleitete studentische Forschen, etwa in Form von Evaluationen, Dienstleistungen für Dritte erbringt, kann es die Politikwissenschaft im öffentlichen Raum legitimieren, die Reputation des eigenen Hochschulstandorts steigern und so die zunehmend bedeutende ,dritte Mission“ der Universitäten bedienen. Dass hier versteckte Kosten warten, wenn Lehrende die Erwartungen von Studierenden und Auftraggebern managen müssen, verschweigt der Autor allerdings nicht. Sie dürften erheblich sein.

Aus Sicht der Hochschuldidaktikerin bietet Freise mit dem starken Fokus auf politikwissenschaftliche Studiengänge eine gut nachvollziehbare Begründung, warum sich gerade diese für forschendes Lernen eignen. Das Konzept selbst wird aufbauend auf den Arbeiten Ludwig Hubers gut fundiert und folgt dem aktuellen Stand der hochschuldidaktischen Diskussion. Ein bisschen Mehr diesbezüglich könnte die Anbindung zur Literatur des teaching-research nexus (vgl. Healy, 2005) bieten, obwohl generell auch wichtige Quellen aus angloamerikanischen Kontexten geliefert werden. Dieser theoretische Strang könnte auch stärker die von Freise geführte Diskussion um das Scheitern im forschenden Lernen fundieren, die ohne Frage für den Lernerfolg der Studierenden höchst relevant ist.

\section{6 Übergreifende Einschätzung der Reihe}

Die gemeinsame, kollaborative Betrachtung der Reihe aus Sicht eines politikwissenschaftlich Lehrenden und eines Hochschuldidaktikers ergibt drei übergreifende Aspekte. Das Format unterstützt die Rezeptionsmöglichkeiten weitgehend. Dank der Kürze und Übersichtlichkeit der Bände laden diese zur effizienten Lektüre und Auseinandersetzung mit der eigenen Lehre an und sollten in keinem Regal von (verantwortlich) Lehrenden in der Politikwissenschaft fehlen. Die Themen sind inter- 
essant, aber sicher noch ausbaufähig. Empfohlen für die Vertiefung und Ausweitung sei hier der Blick in das Handbook of Teaching and Learning in Political Science and International Relations (Ishiyama, Miller \& Simon, 2016), das eine Vielzahl weiterer Themen behandelt. Die Art und Weise, wie die Autorin und die Autoren die Themen behandeln, sind zudem recht unterschiedlich. Das spiegelt den Zustand der Zugänge zum Thema Lehren in der Politikwissenschaft wider. Folgt man der positiven Bewertung der Vielfalt, ist auch dies ein Vorteil der Reihe.

Wir wünschen uns gerne mehr Beschäftigungen mit Hochschullehre, die allgemeine didaktische Prinzipien aufgreift und diese in Bezug auf Politikwissenschaft spezifiziert, kritisiert und weiterentwickelt. Die Kleine Reihe Hochschuldidaktik Politik macht hier einen vielversprechenden Anfang. Ihre Fortsetzung und Vertiefung dürfte ebenfalls Anklang finden. Vielleicht tut sich hier ja wirklich etwas.

\section{Rezensierte Literatur}

Gloe, M. (2018). Kompetenzorientierung in der politikwissenschaftlichen Hochschullehre. Frankfurt/Main: Wochenschau Verlag.

Stykow, P. (2018). Prüfen in politikwissenschaftlichen Studiengängen. Frankfurt/ Main: Wochenschau Verlag.

Cronqvist, L. (2018). Wissenschaftliches Schreiben in der politikwissenschaftlichen Hochschullehre. Frankfurt/Main: Wochenschau Verlag.

Freise, M. (2018). Forschendes Lernen in der politikwissenschaftlichen Hochschullehre. Frankfurt/Main: Wochenschau Verlag.

\section{Literatur}

Anderson, L.W., und D.R. Krathwohl. 2001. A taxonomy for learning, teaching and assessing. A revision of bloom's taxonomy of educational objectives. New York: Addison Wesley Longman.

Biggs, J., und C. Tang. 2011. Teaching for quality learning at university. Maidenhead: McGraw-Hill and Open University Press.

Healey, M. 2005. Linking research and teaching exploring disciplinary spaces and the role of inquiry-based learning. In Reshaping the university: new relationships between research, scholarship and teaching, Hrsg. R. Barnett, 30-42. Maidenhead: McGraw-Hill/Open University Press.

Huber, L. 2011. Forschen über (eigenes) Lehren und studentisches Lernen - Scholarship of Teaching and Learning (SoTL): Ein Thema auch hierzulande? Das Hochschulwesen 4:118-124.

Ishiyama, J., W.J. Miller, und E. Simon (Hrsg.). 2016. Handbook on teaching and learning in political science and international relations. Cheltenham: Edward Elgar Publishing.

Jenkins, S. 2015. Flipping the introductory American politics class: student perceptions of the flipped classroom. PS: Political Science \& Politics 48(4):607-611. 